\title{
Editorial: Combined Modality Treatment of Resectable and Borderline Resectable Pancreas Cancer: Expert Consensus Conference
}

\author{
Jordan Berlin, MD ${ }^{1}$, John P. Hoffman, MD, FACS ${ }^{2}$, and William F. Regine, MD ${ }^{3}$ \\ ${ }^{1}$ Vanderbilt-Ingram Cancer Center, Nashville, TN; ${ }^{2}$ Fox Chase Cancer Center, Philadelphia, PA; ${ }^{3}$ University of Maryland \\ Medical Center, Baltimore, MD
}

The authors describe the current issues in the adjuvant and neoadjuvant treatment of both clearly resectable and borderline resectable adenocarcinomas of the pancreas. After decades of conducting research in the treatment of localized pancreas cancer, few trials have been conducted and even fewer answers are available.

The consensus statement by Abrams et al. ${ }^{1}$ focuses on patients who are considered to have localized pancreas cancer, but, in fact, while pancreas cancer may appear localized on radiographic exam, most patients actually have undetected systemic disease. Evidence for this comes from recent adjuvant therapy trials in which those patients who had R0 or R1 resections still had mortality rates of $\sim 90 \%$ at 5 years when treated with surgery alone (ESPAC-1 and CONKO-001). ${ }^{2,3}$ The fact that current adjuvant therapy may double the survival rates at 5 years provides little comfort to the patients and their families since approximately $80 \%$ of these "resectable" patients still do not survive this disease. To be prepared for a time when more effective systemic options exist, the authors of the consensus article look at the best steps currently available.

First, to make results from various trials easier to interpret, the language and conduct of clinical trials needs to improve. In the postoperative, adjuvant setting it is important that definitions of margin status are consistent

Proceedings of the Consensus Conference sponsored by the American Hepato-Pancreato-Biliary Association and co-sponsored by The Society for Surgery of the Alimentary Tract and The Society of Surgical Oncology, The Gastrointestinal Symposium Steering Committee held in Orlando, Florida, January 24, 2008.

(C) Society of Surgical Oncology 2009

Published Online: 24 April 2009

J. Berlin, MD

e-mail: jordan.berlin@vanderbilt.edu between trials and between institutions. For example, the number of margins assessed may vary and how many slides are evaluated at each site may differ. Institutions may take alternative approaches to inking, or marking, the tumor margins, which can be a difficult task in a resected specimen. Different trials have significant variations on eligibility criteria, definitions of radiation ports, technique for radiation, and criteria for supportive care of the pancreas cancer patient, all of which can affect outcome of the trials. Without a common language for and approach to clinical trials, new studies will continue to face serious criticism, and more decades will pass without significant improvements in patient care.

To improve upon the current options for localized disease, a multidisciplinary approach is clearly necessary. However, one key element to this approach, systemic therapy, has not been effective in advanced disease. Gemcitabine, the current standard cytotoxic agent, is minimally effective in controlling disease, and the targeted agents that have yielded hope in other cancers have provided little benefit in metastatic pancreas cancer. In fact, since the approval of gemcitabine, only one systemic regimen, gemcitabine + erlotinib, has had a statistically significant benefit for survival compared with gemcitabine alone. ${ }^{4}$ However, there are still questions as to the clinical significance of the survival benefit provided by adding erlotinib to gemcitabine. Thus, researchers are left with limited options for the next systemic step to improve control of disease. Whether or not to add erlotinib to gemcitabine in the adjuvant setting is one of the key questions being asked by the currently planned intergroup adjuvant study designed by the Radiation Therapy Oncology Group (RTOG) and the Intergroup Task Force on Pancreas Cancer.

Based on the results of the trials reviewed, it is clear that patients with resected pancreatic adenocarcinoma have 
improved survival with adjuvant therapy. ${ }^{2,3,5}$ Compared with no adjuvant therapy, systemic therapy resulted in improved survival in both the ESPAC-1 trial and the CONKO-001 trial. ${ }^{2,3}$

The consensus statement is somewhat equivocal about the results of RTOG 9704 relating to the primary endpoint of survival in patients with pancreatic head carcinomas. ${ }^{5}$ They correctly state that gemcitabine administered before and after 5FU-based chemoradiation resulted in a statistically significant improvement in overall survival on multivariate analysis (at $P=.05$ ). The sentence following this in the consensus statement reads "this survival difference was not statistically significant." It should read "this survival difference was not statistically significant on univariate analysis." On a preplanned multivariate analysis, the results for survival in patients with cancer of the head of the pancreas reach statistical significance with a $P$ value of exactly .05 . It is important to note that the study clearly defined statistical significance as a $P$ value of $\leq .05$. The multivariate analysis provides a more precise estimate of the treatment effect, given that it adjusts for known prognostic variables.

When controlling for patients with a low postresection serum CA 19-9, as used in CONKO-001, the gemcitabine arm of RTOG 9704 had a 2-month longer median survival compared with CONKO-001, with the caveat that this is comparing trials with very different designs. Moreover, data from institutional trials, as well as retrospective analysis and SEER analysis all suggest an improvement in both disease-free and overall survival with the use of radiation in the adjuvant setting. The more recent CONKO001 and the RTOG 9704 studies suggest the superiority of a gemcitabine-based chemotherapy over a 5-FU based regimen. Analysis of RTOG 9704 also demonstrated the lowest local recurrence rate $(23 \%)$ of any previously reported Phase III adjuvant trial to date (previous range, $37 \%-63 \%$ ). This result may be attributable to the fact that RTOG 9704 was the first Phase III adjuvant trial to require prospective quality assurance (QA) of radiation therapy (RT) planned for patients on study. In addition, review of the data from RTOG 9704 demonstrated that RT delivery "per protocol" had a significant impact on patient survival and potential toxicity. ${ }^{6}$ The effects of radiation are encouraging, but as yet they remain to be absolutely proven. In Europe, a recent randomized Phase II study was completed comparing gemcitabine alone with gemcitabine plus radiation, but this will not proceed to a Phase III trial because of a lack of funding. The second important question being asked by the planned RTOG study (0848) to be conducted in the United States, Canada, and Europe is whether or not chemoradiation at the end of chemotherapy provides survival benefit. Ironically, presuming the successful completion of the RTOG 0848 trial, if radiation improves local control, but not survival, we may have to revisit the radiation therapy question when better systemic control is finally achieved.

It is also time to explore some options beyond surgery followed by postoperative therapy for the localized pancreas cancer patient. Neoadjuvant approaches, while as yet unproven by a Phase III trial, should figure prominently in future clinical trials. The rationale for a neoadjuvant approach has been enumerated by many. First, pancreas cancer surgery has significant risk for morbidity that may prevent patients from receiving postoperative treatment with either chemotherapy or chemoradiotherapy. A neoadjuvant approach would allow a higher percentage of patients to receive multimodality treatments. Second, an effective neoadjuvant approach should theoretically improve the chances at achieving an R0 resection. Third, with tumor still in place, smaller radiation fields may be designed that theoretically will result in less short- and long-term morbidities from radiation. Finally, it is theoretically possible that a neoadjuvant approach will "weed out" the patients with the most aggressive, treatmentresistant disease. These patients would progress during the neoadjuvant treatment period and prevent them from unnecessarily undergoing surgery and its attendant risks.

Unfortunately, current neoadjuvant therapy results are limited to single-institution, nonrandomized studies. Because patient selection can play a crucial role in Phase II treatment results, multi-institutional studies with common language and eligibility requirements are necessary. Investigators will have to determine the best approach to define potential resectability. Is triphasic computed tomography (CT) scan with thin cuts alone sufficient? Should endoscopic ultrasound (EUS) be used for anything more than biopsy? Do we need preoperative laparoscopy to rule out peritoneal disease that is not well detected by any current radiographic techniques? Next, the definitions of resectable and borderline resectable need to be made more consistent. Many issues remain controversial, such as: how much of the superior mesenteric or hepatic artery can be surrounded on CT before a tumor is deemed borderline resectable or unresectable, or whether or not the need to replace a segment of vein defines a resectable or borderline resectable status.

With parallel study of both neoadjuvant and postoperative adjuvant approaches, treatment sequencing might become an important future issue of study. A prospective, randomized trial comparing preoperative with postoperative therapy would not be easy. Patients would need to register and undergo laparoscopy before randomization, thus obliging surgeons to agree to participate and to discuss the alternatives. Those developing systemic metastases during neoadjuvant therapy and those found at both the surgery-first and surgery-second arms to have unresectable 
or metastatic disease would need to be accounted for in the statistical design. Qualify of life (QOL) and cost analyses would be important secondary endpoints of such a study. Most importantly, this question has been difficult to ask in the past with only one of three trials asking the same type of question successfully completing accrual in rectal cancer.

Finally, the authors of the consensus article also address one of the most difficult issues in localized pancreas cancer: the recently identified category of borderline resectable patients. ${ }^{1}$ For years, physicians treating pancreas cancer have largely ignored the TNM staging to place patients in three categories: resectable, locally advanced but unresectable, and metastatic. Now, with better radiographic techniques and a renewed focus on surgeons with expertise in pancreas cancer surgery, a fourth category has been defined radiographically. These patients need to be consistently identified a priori as the goals of therapy differ from the truly unresectable patients where shrinkage, no matter how great, will not change the ability to perform surgery (for example, the completely encased superior mesenteric artery). We concur with the authors of the consensus statement that definitions need to be uniform, consistent, and clear. These patients need their own trials that have endpoints such as treatment response and an increase in the margin-negative resectability rate. The ultimate goal in performing such studies is to determine if in producing responses and/or improving $\mathrm{R} 0$ resection rates, some patients are cured or can live disease- and symptom-free for longer periods of time. If these patients with intermediate prognoses are allowed into trials for patients with either clearly resectable or clearly unresectable cancers, they should be identified, as their presence in either cohort could affect the study results.

There are some areas where the guidelines for defining borderline resectable disease as outlined in the consensus statement, right or wrong, may not have gone far enough. First, it is clear that not all CT scanners are of the same quality. While few, if any, nonspiral CT scan machines exist within the United States, the number of slices obtained at a time can still differ, and as technology continues to move forward, the number of millimeters between each slice may also change, improving the ability to see the vessel/tumor interface. Three-dimensional reconstructions can be very helpful to the surgeon in determining resectability, but this technique is not applied in every case or at every institution. Finally, the expertise of the radiologists, like the expertise of all physicians involved in the care of pancreas cancer, is not uniform, and this variability may lead to differences in the approach to the same patient at multiple institutions. More work is needed to precisely define what degree of portal/SMV impingement is consistent with borderline resectability. Variability may also be a significant problem with the surgeons. As unpleasant as it is to note that not all physicians have the same level of skills, multiple papers suggest that practice is important with regard to the pancreatoduodenectomy. Physicians and institutions with high volumes appear to have better outcomes than physicians and institutions with low volumes for pancreas cancer. ${ }^{7}$ However, the majority of patients do not have their care given by high-volume surgeons for practical reasons, and this problem does not lend itself to an easy solution. However, in truly moving forward with the care of these patients it is impossible to ignore these data as it remains so consistent.

Finally, there are undoubtedly one or more biologic factors that will more precisely define a group intermediate to the resectable and unresectable than purely radiologic parameters. Until they are discovered, radiologic tools are the ones we must use and perfect in order to prescribe optimal therapy.

In conclusion, the consensus article provides guidelines upon which we can develop trials for treating localized pancreas cancer. ${ }^{1}$ With a common approach to this deadly disease we will have a better chance to succeed in the future.

\section{REFERENCES}

1. Abrams, RA, Lowy AM, O'Reilly EM, Wolff RA, Picozzi VJ, Pisters PWT. Combined modality treatment of resectable and borderline resectable pancreas cancer: Expert consensus statement. Ann Surg Oncol. 2009; [Epub ahead of print]. DOI:10.1245/ s10434-009-0413-9.

2. Neoptolemos J P,Stocken DD, Friess H, Bassi C, Dunn JA, Hickey $\mathrm{H}$, et al. A randomized trial of chemoradiotherapy and chemotherapy after resection of pancreatic cancer. $N$ Engl $\mathrm{J}$ Med. 2004;350:1200-10.

3. Oettle H, Post S, Neuhaus P, Gellert K, Langrehr J, Ridwelski K, et al. Adjuvant chemotherapy with gemcitabine vs observation in patients undergoing curative-intent resection of pancreatic cancer: a randomized controlled trial. JAMA. 2007;297:267-77.

4. Moore MJ, Goldstein D, Hamm J, Figer A, Hecht JR, Gallinger S, et al.: Erlotinib plus gemcitabine compared with gemcitabine alone in patients with advanced pancreatic cancer: a phase III trial of the National Cancer Institute of Canada Clinical Trials Group. J Clin Oncol. 2007;25:1960-6.

5. Regine WF, Winter KA, Abrams RA, Safran H, Hoffman JP, Konski A, et al. Fluorouracil vs gemcitabine chemotherapy before and after fluorouracil-based chemoradiation following resection of pancreatic adenocarcinoma: a randomized controlled trial. JAMA. 2008;299:1019-26.

6. Abrams RA, Winter KA, Regine WF, Safran H, Hoffman JP, Konski AA, et al. RTOG 97-04-Radiotherapy Quality Assurance (QA) Review and Survival. Proceedings of ASTRO, Int J Radiat Oncol Biol Phys. 2006;66:S22.

7. Lieberman MD, Kilburn H, Lindsey M, Brennan MF. Relation of perioperative deaths to hospital volume among patients undergoing pancreatic resection for malignancy. Ann Surg. 1995;225:638-45. 